\title{
Mast cell response to Leishmania mexicana and sand fly salivary proteins is modulated by androgens
}

\author{
Laura Sánchez-García ${ }^{1 *}$, Armando Pérez-Torres², Samira Muñoz-Cruz ${ }^{3}$, Jorge Morales-Montor ${ }^{4}$ and Ingeborg \\ Becker'.
}

1 Unidad de Investigación en Medicina Experimental, Centro de Medicina Tropical, Facultad de Medicina Universidad Nacional Autónoma de México, Avenida Universidad 3000, C.P. 04510, Ciudad de México, México.

2 Departamento de Biología Celular y Tisular, Facultad de Medicina, Universidad Nacional Autónoma de México, Ciudad de México, México.

3 Unidad de Investigación Médica en Enfermedades Infecciosas y Parasitarias. Centro Médico Nacional Siglo XXI. Instituto Mexicano del Seguro Social. México.

4 Departamento de Inmunología, Instituto de Investigaciones Biomédicas, Universidad Nacional Autónoma de México, AP 70228, Ciudad de México, 04510, México

* Corresponding authors: Telephone + (5255) 56223854, + (5255) 56223732; Fax number: + (5255)56223369. Emails: laurasan@uqroo.edu.mx; laurasanchezg@hotmail.com

Abstract: Mast cells (MCs) play a crucial role during infections with Leishmania, that is transmitted through the bite of an infected sand fly that injects saliva together with the parasite. Sand fly saliva is a complex fluid that modulates the host immune response. In addition, hormonal factors modulate the host immune response, impacting the susceptibility to infections. Thus, to assess the impact of androgens and salivary proteins of sand fly vectors on the mast cell (MC) response to Leishmania infections, we infected orchiectomized male mice with the parasite in the presence or absence of sand fly salivary proteins and analyzed the inflammatory response of MCs. Our results showed a differential MC response to the parasite and to vector salivary proteins in mice deprived of gonadal hormones, as compared to sham-operated mice. Orchidectomy induced a different pattern of activation in MC of animals infected with Leishmania and vector-salivary proteins. Our results show that during Leishmania infection, androgens modulate the innate immunity response against the parasite and salivary proteins of the sand fly vector. 


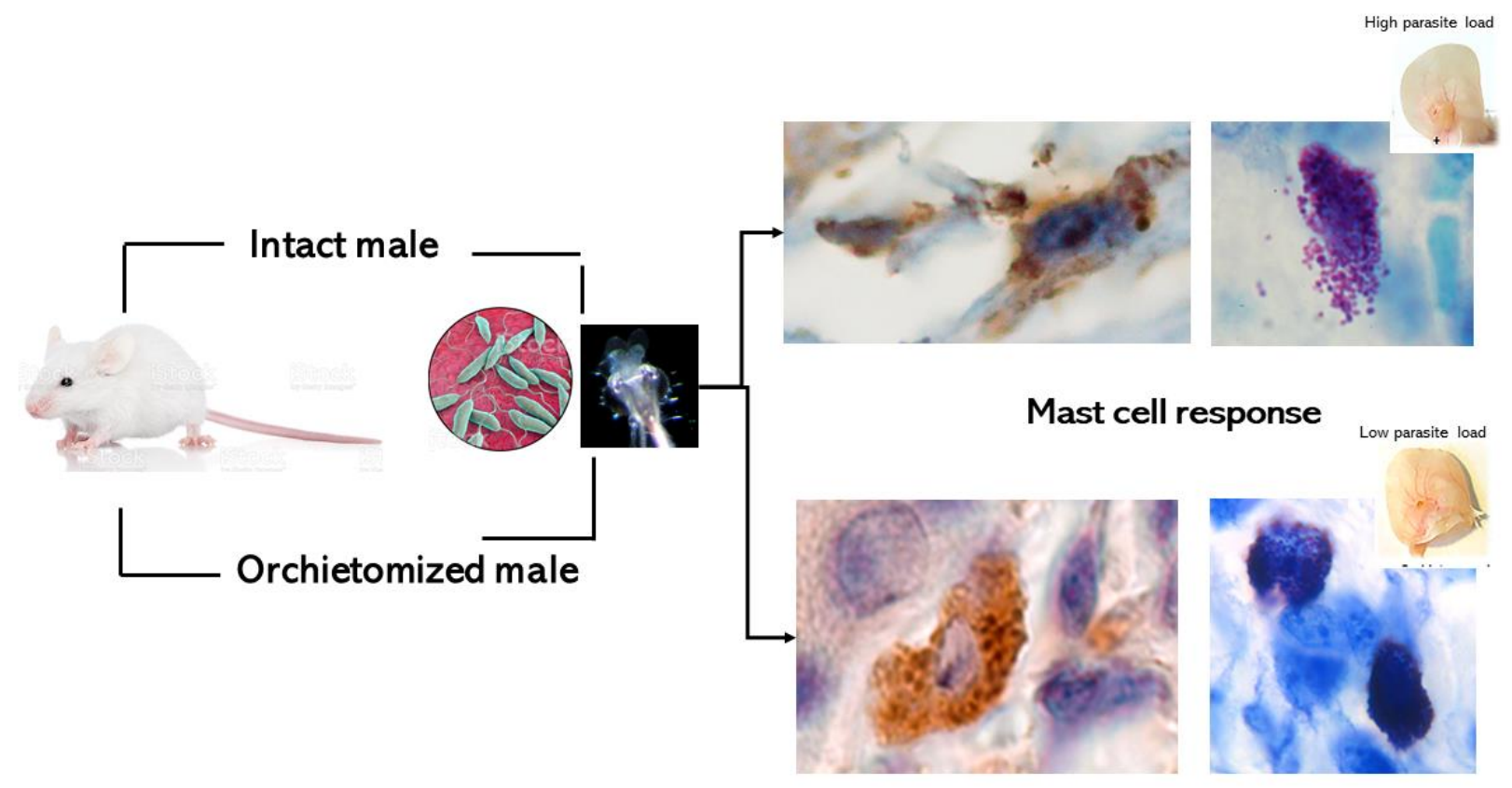

Keywords: Mast cells, Leishmania mexicana, sand fly salivary proteins, sexual hormones

\section{Introduction}

Mast cells (MCs) are considered sentinels of the innate host defense due to their ability to detect pathogens and degranulate immediately upon activation. They are strategically distributed throughout most tissues of the body and possess the capacity to differentiate into life-long sessile tissue homing cells [1,2,3], making them unique. Mast cells mediated their physiological and pathophysiological roles through the selective release of various preformed and newly synthesized inflammatory mediators $[4,6]$. These include different proteases, histamine, heparin proteoglycan, chondroitin sulfate E, acidic hydrolases, and various cytokines and growth factors $[4,6]$. However, the role of activated MC in response to infections remains controversial. It is considered a double-edged sword: on the one hand, specific mediators can contribute to contain or eliminate the pathogen, yet they can also favor the progression of infections by diminishing or augmenting the intensity of inflammation, depending on the pathogen, age, gender, and the genetic background of the host [4,5]. Leishmania infections (both in vitro and in vivo) have shown that MC degranulation influences the early inflammatory response, thereby contributing to the outcome of the infection, which also depends on the genetic background of the host $(7,8,9)$. Thus, in BALB/c mice, MCs seem to facilitate disease progression, while in C57BL/6 mice, MCs promote disease control [7].

Leishmania transmission occurs through the bite of an infected female sand fly during blood feeding, which injects the parasite together with salivary components [10,11]. After the infection, both the parasite and proteins of the sand fly saliva affect the immune response of the host [12].

Furthermore, the immune system is also strongly influenced by the endocrine system since sex hormones modulate and regulate the differentiation, proliferation, and activation of immune cells $[13,14]$.

Gender also modulates Leishmania infections $(15,16,17,18,19)$. Thus, estrogens upregulate IL-4 and IL-10 production, whereas testosterone downregulates IL-2, IL-6, TNF-a, and nitric oxide production in macrophages. 
During L. donovani infections, testosterone down-regulates p38 MAPK activation, inhibiting the macrophage inflammatory and microbicide response [20,21,22]. Additionally, dihydrotestosterone (DHT) increases Leishmania mexicana (L. mexicana) infectivity, both in vivo and in vitro [23].

Yet, the regulation exerted on MC by endocrine factors, Leishmania parasites and vector salivary proteins during acute and chronic infections, remain to be analyzed. Therefore, the aim of the current study was to analyze MC activation in male BALB/c mice deprived of gonadal hormones and infected with L. mexicana, in the presence and absence of sand fly-salivary proteins.

\section{Results}

2.1. Ear tissue histology after inoculation of L. mexicana and vector salivary proteins.

To assess MC activation in the presence or absence of male sexual hormones after L. mexicana and salivary gland lysate (SGL) inoculation, the ears of intact, sham, and orchiectomized BALB/c male mice were analyzed by histology. After the inoculation of L. mexicana and vector salivary lysate, intact BALB/c male mice showed a dermal inflammatory response, started at 30 minutes after the challenge and remained from 8 to 72 hours, characterized by neutrophilia (Figure A-E black arrows). Orchiectomized mice also showed edema, vascular congestion, and vasodilatation but a less neutrophilia and dermal neutrophil infiltration (Figures 1F-H, arrows) than intact mice between 30 min and 24 hours after inoculation. Interestingly, a delay of 48 hours was observed in the recruitment of neutrophils in orchiectomized mice (Figure 1I). In addition, as compared to sham mice (Figure 1I) the intense neutrophil infiltration in the orchiectomized mice occurred between 48 to 72 hours (Figure 1 I-J).

A

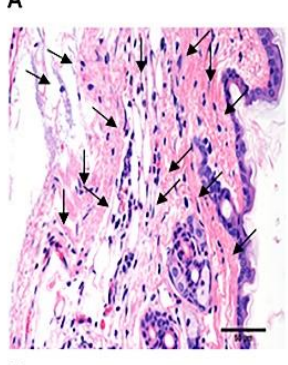

$\mathrm{F}$

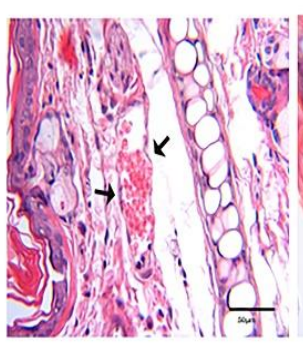

B

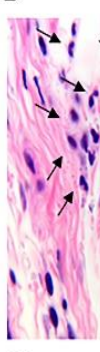

G

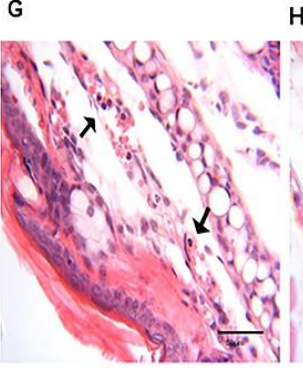

$\mathrm{H}$
C<smiles>[2H]C1CCCC1</smiles>
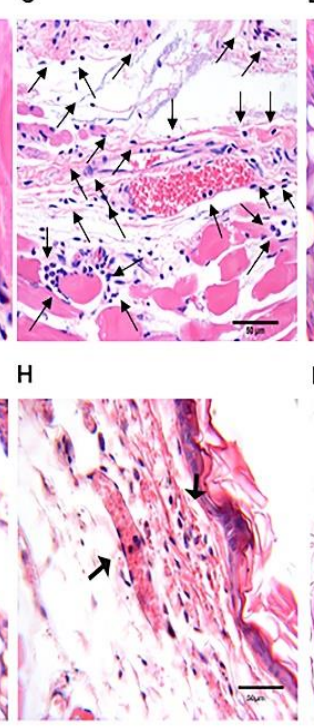

\section{D}

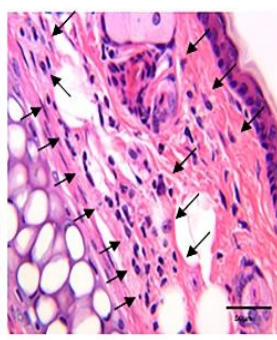

I

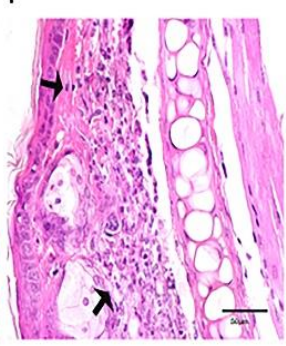

E

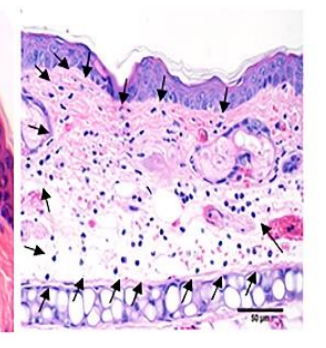

J

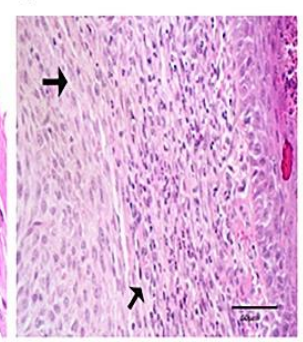

Figure 1. Comparative histological images of kinetics of the inflammatory cell infiltration of sham and orchiectomized BALB/c mice, in response to L. mexicana together with salivary gland lysates (SGL). Hematoxylin and eosin-stained ear sections of sham-operated (A-E) and orchiectomized mice (F-J), at 30 min. (A \& F), 8 h (B \& G), 24 h (C \& H), 48 h (D \& I), and $72 \mathrm{~h}$ (E \& J), following inoculation with L. mexicana and SGL. In sham mice, accumulation of inflammatory cells, principally neutrophils, is evident at 30 minutes after challenge (A), and remains intense up to $48 \mathrm{~h}$ (D), weakening thereafter at $72 \mathrm{~h}(\mathrm{E})$. In contrast, in orchiectomized mice, the neutrophil infiltration is delayed, beginning after $24 \mathrm{~h}$ after challenge $(\mathrm{H})$ and remaining intense throughout $48 \mathrm{~h}(\mathrm{I})$ and $72 \mathrm{~h}(\mathrm{~J})$. Original magnification $\times 40(\mathrm{~A}-\mathrm{I})$ and $\times 10(\mathrm{~J})$. Scale bar $=50$ um Photomicrographs are representative of three independent experiments with five replicates for each group. Black arrows show neutrophils within venules and capillaries 
Furthermore, MC number recruitment and degranulation also showed slight differences between orchiectomized and sham mice. A slightly higher increase in MC infiltration was observed in gonadectomized mice, as compared to sham mice, at all time points (Figure 2).

A

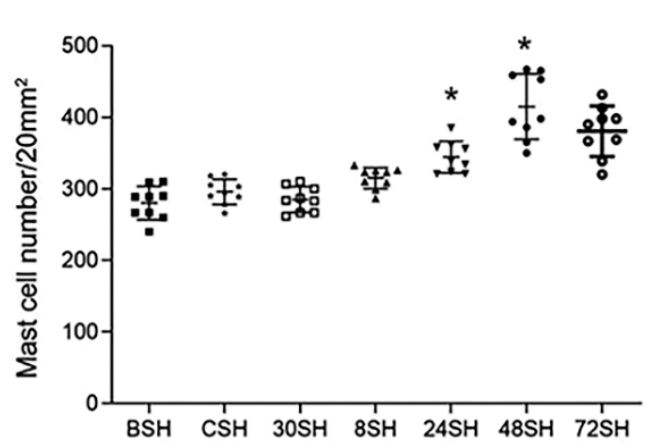

B

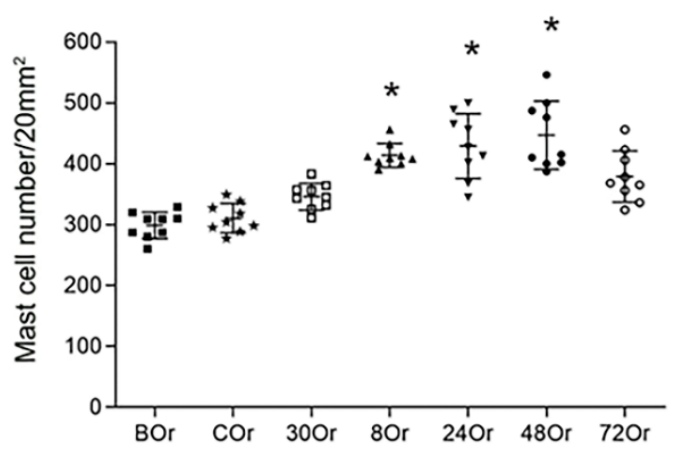

Figure 2. MCs degranulation in sham and orchiectomized mice throughout $72 \mathrm{~h}$. Total mast cell numbers were counted in toluidine blue-stained ear sections from sham-operated (A) and orchiectomized mice (B), at $30 \mathrm{~min}, 8 \mathrm{~h}, 24 \mathrm{~h}, 48 \mathrm{~h}$, and $72 \mathrm{~h}$, following inoculation with L. mexicana together with SGL. Counting was done by analyzing the photomicrographs of the total number of mast cell in $20 \mathrm{~mm} 2$. Three independent experiments with five replicates for each group were done. $\mathrm{A}^{*}$ significant differences were found between $\mathrm{BSH}, \mathrm{CSH}, 30 \mathrm{SH}$ and $8 \mathrm{SH}$ vs $24 \mathrm{SH}$ and $48 \mathrm{SH}$. $\mathrm{B}^{*}$ significant differences were found between BOr, COr, 30Or vs 8Or, 24Or and 48Or. Nonparametric ANOVA ordinary multiple comparison was employed (Significance $\mathrm{p}<0.0001$ ). (Abbreviations: BSH: basal sham; CSH: control sham; 30SH: 30 min sham; 8SH: 8 h sham; 24SH: 24 h sham, 48SH: 48 h sham; 72SH: 72 h sham. BOr (basal orchiectomized; COr control orchiectomized; 30r: 30 min orchiectomized, 8-72 Or: 8 -72 h orchiectomized).

After L. mexicana and salivary lysate inoculations, MC recruitment started at 30 min until 48 hours, showing a similar pattern between orchiectomized and sham mice (Figure 2). However, sham mice showed slightly lower numbers of MC throughout all the time points, as compared to orchiectomized mice. In addition, cell counts in orchiectomized mice showed a higher standard deviation compared with sham mice. At 72 hours, no MC increase was observed in either group of mice (Figure 2).

In sham mice, $\mathrm{MC}$ numbers increased after $30 \mathrm{~min}$ and throughout 48 hours compared to basal values $(\mathrm{BSH})(\mathrm{p}<0.001)$. However, after 72 hours, a reduction in mast cell numbers was observed $(\mathrm{p}<0.0001)$.

In orchiectomized mice, a significant increase in MC numbers was registered from 8 to 48 hours ( $p<0.001)$, as compared to basal values, control, and $30 \mathrm{~min}$ (BOr, COr, 30Or). This significant increase was registered after 8 to 48 hours $(p<0.001)$. No significant differences were observed when comparing sham and orchiectomized mice.

It is noteworthy that in orchiectomized animals, MC degranulation revealed a discrete group of granules at 30 min and 48 hours. However, at 72 hours, MC degranulation in orchiectomized animals was similar to that observed in sham mice at 30 min and 8 hours (Figure 3 A-B, J). Whereas in orchiectomized mice, MC degranulation resembled a "piecemeal process," in which the contents of the released granules were surrounded by plasma membranes (Figure 3G-H), in sham mice, the MC degranulation occurs more diffusely ("systematic mode") resembling activation after FceRI high-affinity receptor binding (Figure 3A-E). 


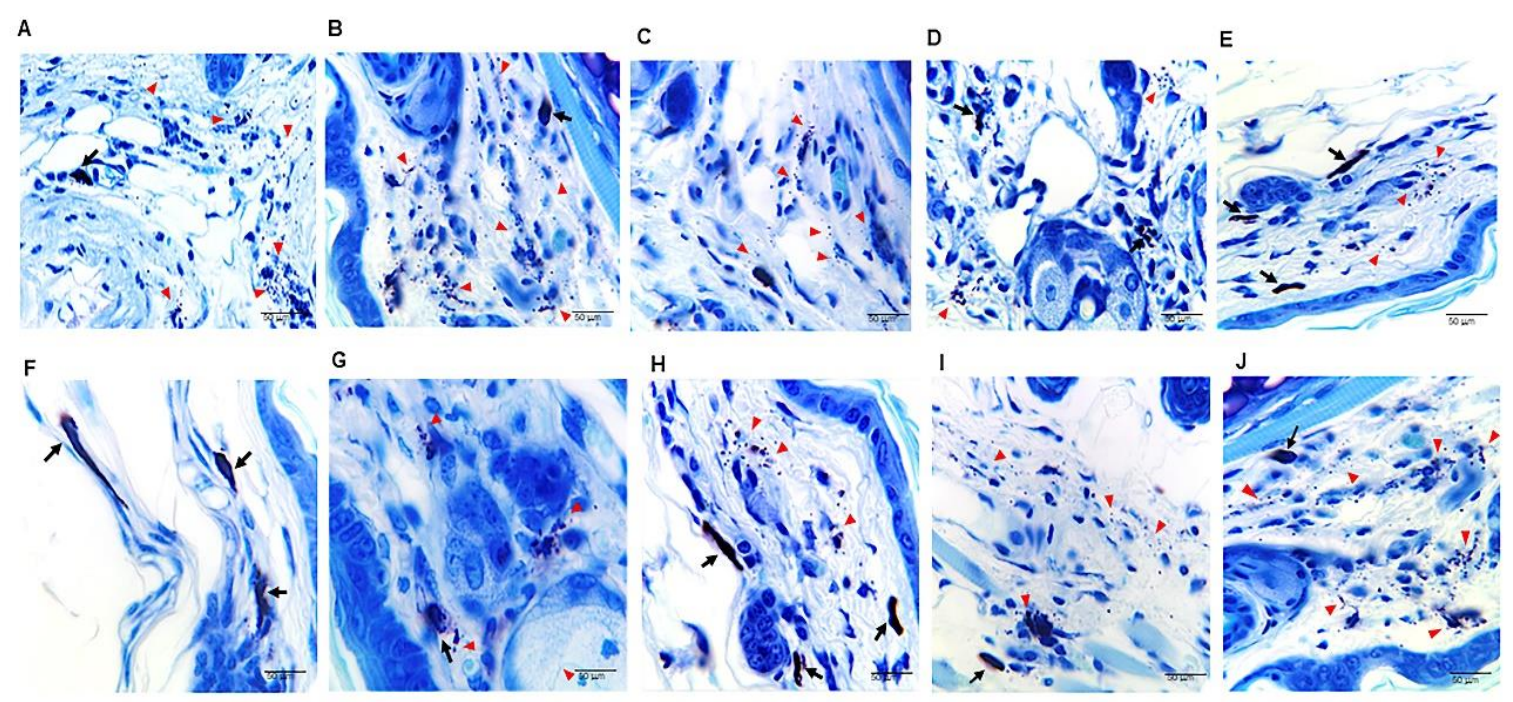

Figure 3. Photomicrographs of mast cells degranulation in ear skin from sham and orchiectomized mice after inoculation with L. mexicana together with salivary gland lysates (SGL). Toluidine blue-stained ear sections from shamoperated (A-E) and orchiectomized mice (F-J), at $24 \mathrm{~h}$ (C \& H), 48 h (D \& I), and $72 \mathrm{~h}$ (E \& J) respectively, following inoculation with L. mexicana together with SGL. Note the intact dark purple mast cells (black arrows) and degranulated mast cells, and mast cell-released dark purple insoluble granular particles (red arrowheads). In sham mice, a mild and minimal mast cell degranulation is detected at $24 \mathrm{~h}(\mathrm{C} \& \mathrm{H})$ and $48 \mathrm{~h}(\mathrm{D} \& \mathrm{I})$ after challenge, whereas at $72 \mathrm{~h}$ (E\& J) some mast cells with replenished granules are observed. In orchiectomized mice, mast cell degranulation also initiates at 24 $\mathrm{h}(\mathrm{H})$ but remains intense at $48 \mathrm{~h}(\mathrm{I})$ and it is maintained $72 \mathrm{~h}(\mathrm{~J})$. Images are representative of three independent experiments with five replicates for each group. Scale bar= $50 \mu \mathrm{m}$.

2.2. Immunohistochemistry of ears after inoculation of L. mexicana and vector salivary gland lysates: inflammatory mediators released after $\mathrm{MC}$ activation.

Histamine, TNF- $\alpha$, and tryptase were assessed after MC activation induced by injection of L. mexicana and vector SGL at $30 \mathrm{~min}, 8,24,48$, and 72 hours. Inflammatory mediators released by orchiectomized mice showed differences compared to sham mice. Histamine, TNF- $\alpha$, and tryptase released in orchiectomized mice at 30 min showed a weak mark compared with sham mice (Figure 4), and their mark increases throughout the infection time. At 48 hours, the inflammatory mediator mark increased in intensity but continued to be less intense than in sham mice (Figure 5). However, at 72 hours, the histamine, TNF- $\alpha$, and tryptase, mark in orchiectomized mice increased in intensity, similar to that of sham mice (Figure 6). Therefore, the lack of male sexual hormones seems to reduce and retard MC activation, their release of granules, and their numbers in the tissues (Figures 4-6). 


\section{Orchiectomyzed}

$30 \mathrm{~min}$ Histamine

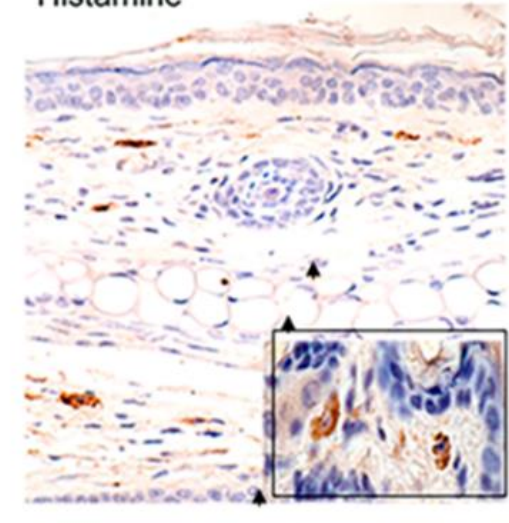

Sham

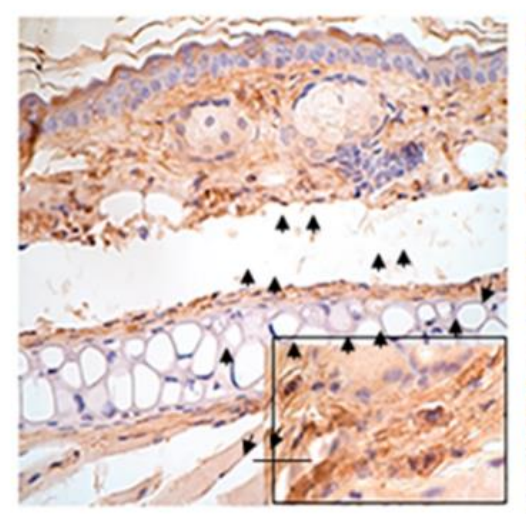

Tryptase
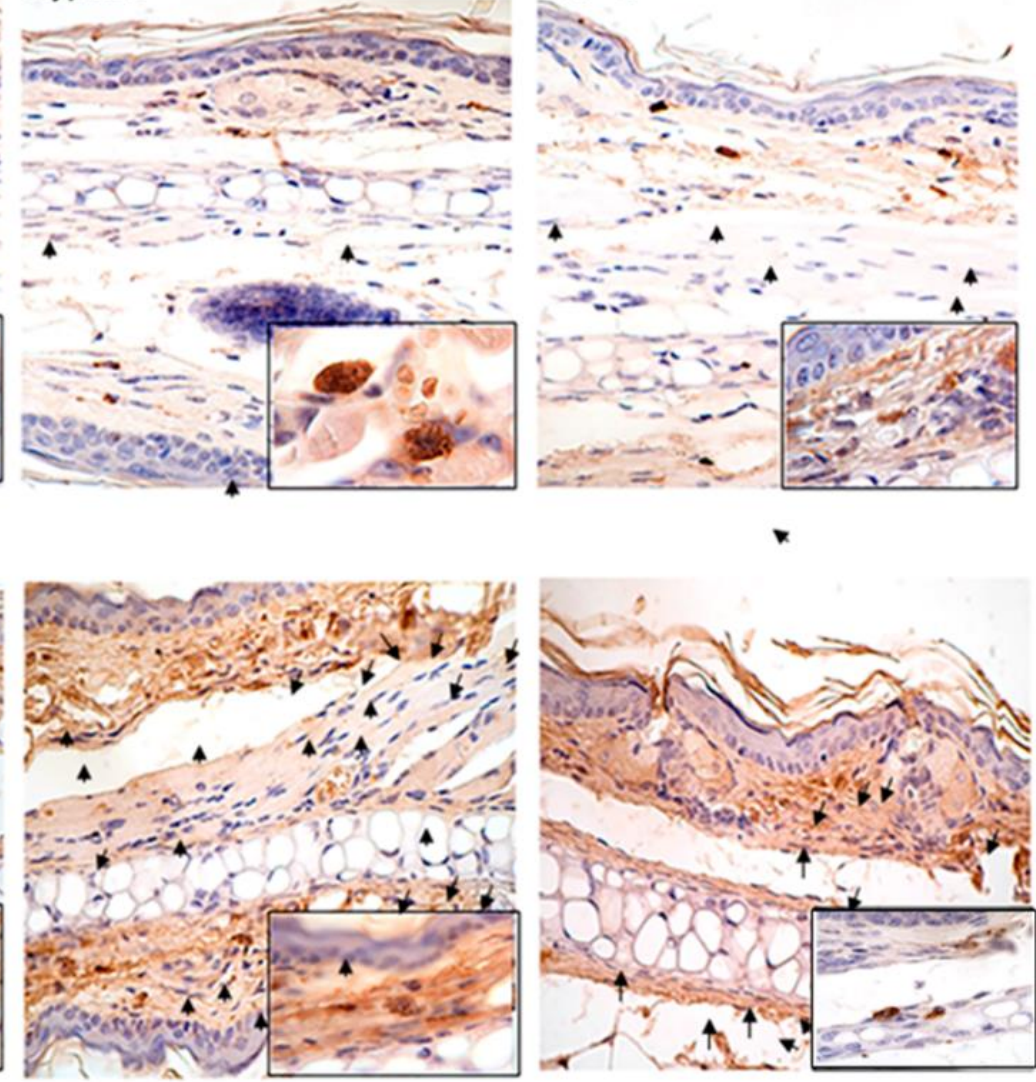

Figure 4. Comparative immunohistochemical assessment of mast cell release of histamine, tryptase and TNF- $\alpha$ in sham and orchiectomized BALB/c mice, at $30 \mathrm{~min}$ in response to the injection of L. mexicana combined with salivary gland lysate. Photomicrographs show dark brown mast cells (black arrows) in sham and orchiectomized BALB7c mice. In dermis from sham mice degranulation and diffuse immunoreactivity are evident at 30 minutes after challenge. Scale bar $=20 \mu \mathrm{m}$. 


\section{Orchiectomyzed}

\section{8 hours Histamine}

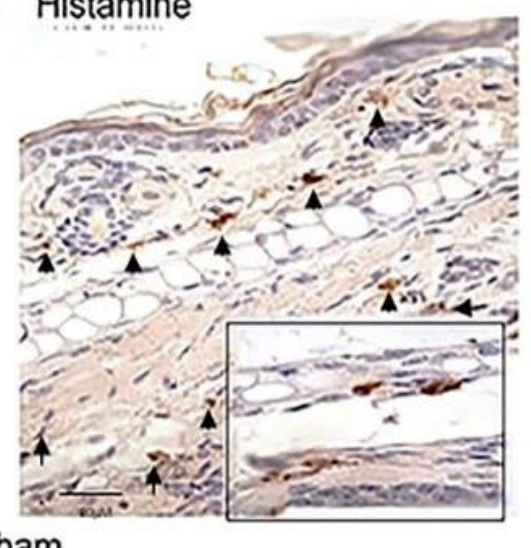

Sham

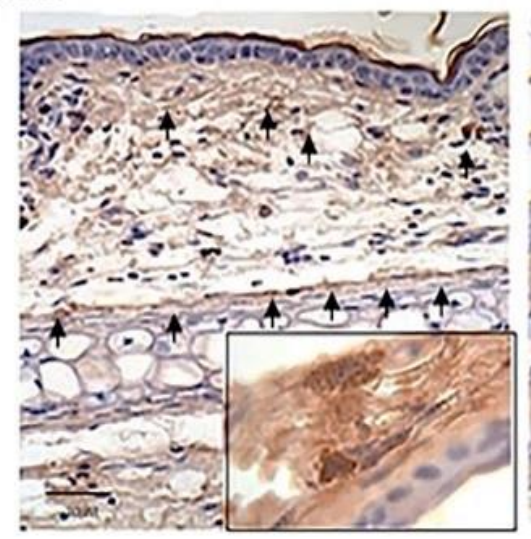

Tryptase
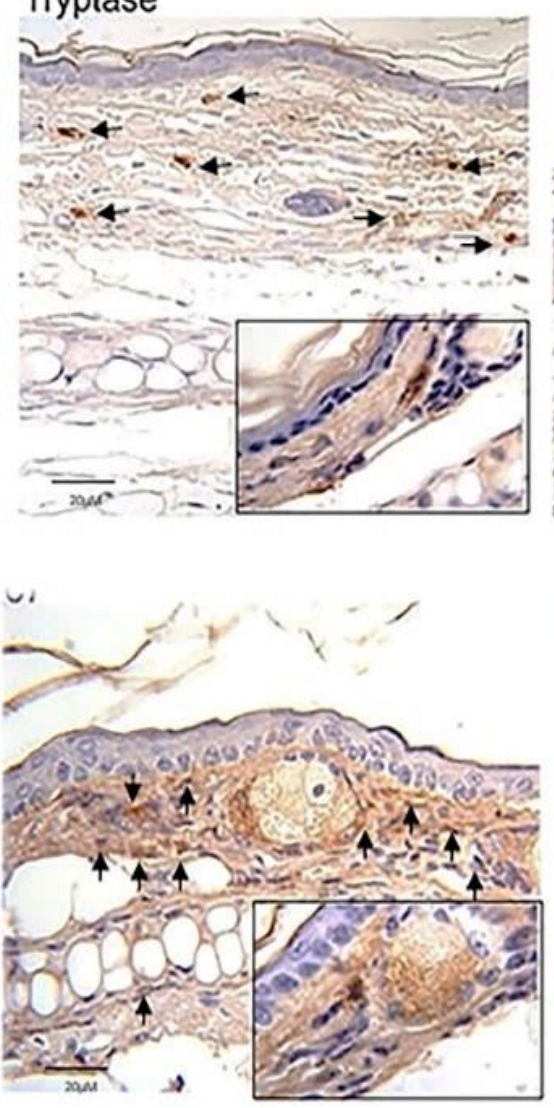
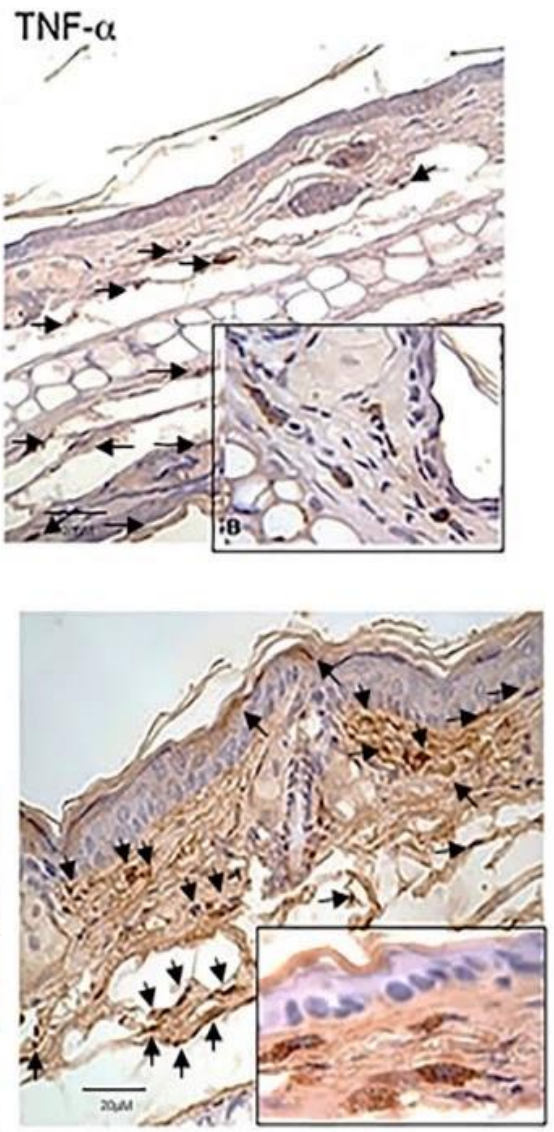

Figure 5. Comparative immunohistochemical assessment of mast cell release histamine, tryptase and TNF- $\alpha$ in sham and orchiectomized BALB/c mice, at 48 hour after L. mexicana and salivary gland lysate injections Photomicrographs show intact dark brown mast cells (black arrows). In dermis from sham mice degranulation and diffuse immunoreactivity are evident at 48 hours after challenge. Images are representative of three independent experiments with five replicates for each group. Scale bar $=20 \mu \mathrm{m}$. 


\section{Orchiectomyzed}

72 hours
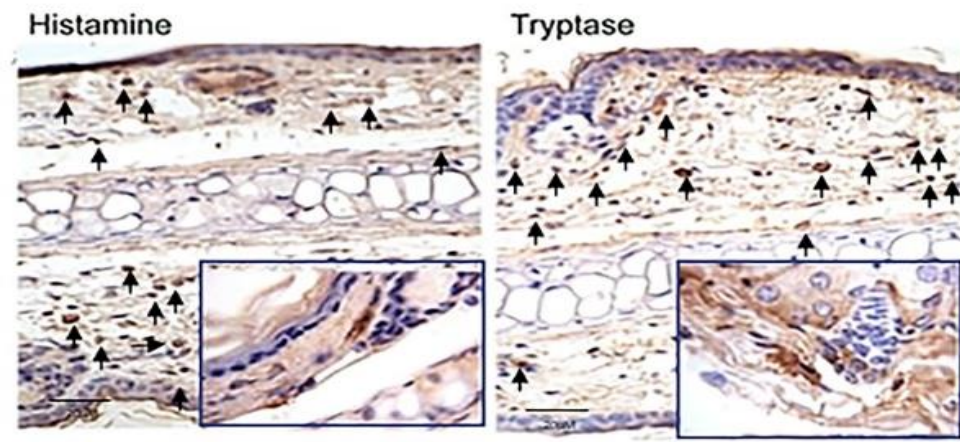

TNF- $\alpha$

Sham
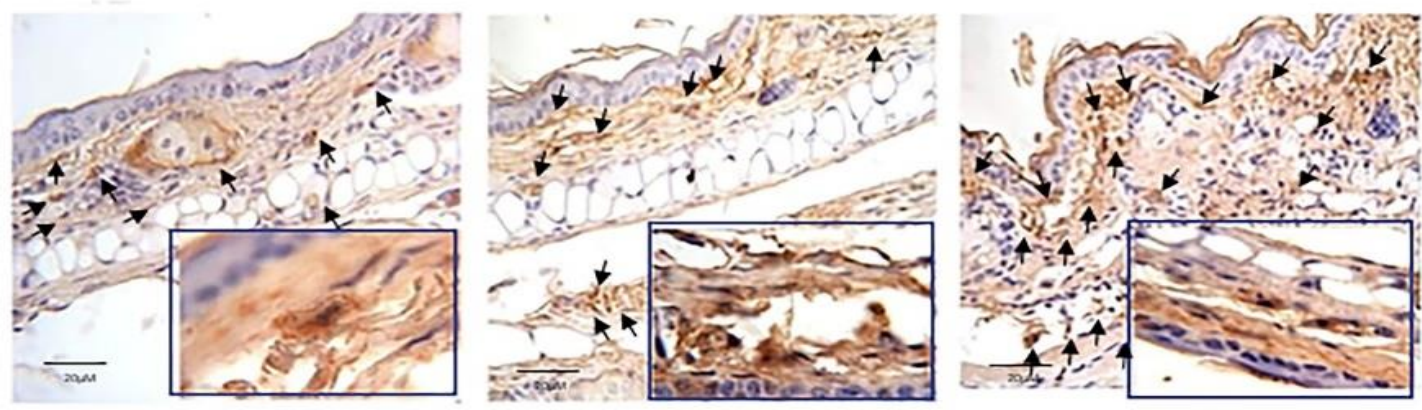

Figure 6. Comparative immunohistochemical assessment of mast cell release histamine, tryptase and TNF- $\alpha$ in sham and orchiectomized BALB/C mice, at 72 hour after L. mexicana and salivary gland lysate injections Photomicrographs show intact dark purple mast cells (black arrows). In dermis from sham mice degranulation and diffuse immunoreactivity are evident at 72 hours after challenge. Images are representative of three independent experiments with five replicates for each group. Scale bar $=20 \mu \mathrm{m}$.

2.3. Chronic infection of L. mexicana together with vector salivary proteins in orchiectomized BALB/c mice.

Chronic L. mexicana infections (4 and 8 weeks) in orchiectomized mice showed smaller ulcers than sham mice (Figure 7). Furthermore, a significant reduction in the number of amastigotes infecting macrophages was observed in gonadectomized mice compared to sham mice (Figure 7). The significant reduction of infected macrophages observed at eight weeks (50.4\%) indicates that gonadectomized mice have better control of the parasite infection. 


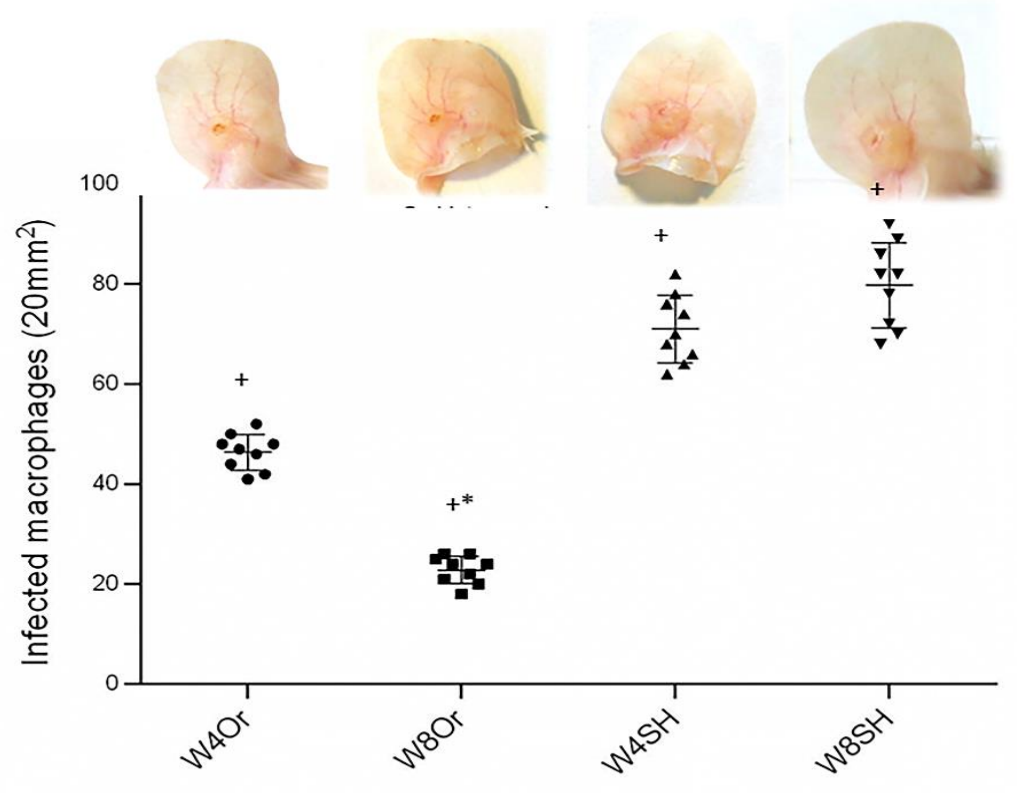

Figure 7. Comparative ulcer size at 4 and 8 weeks after infection and the counting of infected macrophages. L. mexicana promastigotes were inoculated and the chronic infection was analyzed. Orchiectomized mice produced smaller lesions, as compared to sham-operated mice. Low numbers of infected macrophages were evidenced in orchiectomized mice

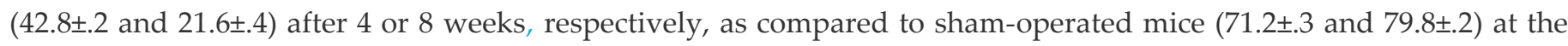
same time points. Orchiectomized mice showed a strict control of the parasite infection at 8 weeks. Images are representative of three independent experiments with five replicates for each group. Significant differences between sham-operated and gonadectomized mice $P \leq .05)$. $\left({ }^{*},{ }^{*}\right)$. Abbreviations: W4Or and W8Or (week 4 and 8 in orchiectomized mice); W4SH and W8SH (week 4 and 8 in sham mice).

\section{Discussion}

The innate and adaptive immune response orchestrated by the endocrine system results in an intricate crosstalk between the different actors involved. This study demonstrated the absence of male hormones produced a delay in the degranulation of mast cells. Gonadectomized male immunological response to L. mexicana and salivary gland proteins shows a tight control of male hormones in developing the parasite infection and the mast cell activation. The chronic L. mexicana infection + salivary glands proteins and delayed mast cell degranulation during acute inflammation result in small cutaneous ulcers and lower parasite load.

It is well accepted that MCs orchestrate the development of the inflammatory process after an infection with Leishmania. Experiments done in vitro have shown that MCs release TNF-a, histamine, tryptase, and IL-1b after contact with the parasite $[26,27,28,29]$. Furthermore, a cause-effect variation in MCs numbers and in the percentage of degranulation has been reported in inoculation sites after L. major infections [24]. Thus, the release of inflammatory mediators, such as TNF-a, tryptase, and histamine is possibly involved in the inflammatory process that ensues after a natural sand fly bite [30]. Furthermore, a pharmacologically induced MC degranulation in susceptible mice was shown to make them more resistant to an L. major infection, with lower parasite loads and decreased lesion sizes [8].

During Leishmania infections in mice, a differential response has been observed according to gender and mouse strain. Previous studies in our laboratory showed a differential MC activation in male mice infected with L. mexicana, which was associated with their resistance to the parasite infection. Susceptibility to L. major and L. mexicana infections is higher in males than females [31,30,32,33]. Fundamental differences between susceptible and resistant mice include MC degranulation and the presence of inflammatory cells. In C57BL/6 males, which are slightly resistant to L. mexicana, a delayed MC degranulation was shown after parasite inoculation, as compared 
to highly susceptible BALB/c male mice [6]. These data seemed to indicate that the delay of inflammatory mediators in C57BL/6 mice favor their control of the infection.

Considering the evidence on the differential response of males to L. mexicana infections, we now analyzed whether the early activation of MC and their release of inflammatory mediators are influenced by male sexual hormones, thereby facilitating the L. mexicana infection. In our infection model we also included sand fly SGL, aiming to simulate natural sand fly infections. Therefore, two groups of mice, orchiectomized and sham, were inoculated with L. mexicana in combination with sand fly SGL. Our results show that in sham BALB/c mice, an early MC degranulation, accompanied by a massive neutrophil infiltration, was evidenced after the inoculation of L. mexicana and vector SGL (Figures 2 and 3). The MC degranulation released inflammatory mediators such as TNF-a, histamine, and tryptase (Figure 4-6), which can contribute to neutrophil recruitment, giving an advantage to the parasites. The non-protective effect of neutrophils has been proposed to resemble that of a "Trojan horse", which shields phagocytosed parasites from extracellular immune mechanisms, permitting their replication within neutrophils. This early evasion strategy seems to facilitate the infection [34,35]. Our data now show that in contrast to sham operated mice, orchiectomized mice showed a discrete and piecemeal mode of MC degranulation and discrete neutrophil infiltration (Figure 3), accompanied by a lower release of inflammatory mediators (Figure 4-6). This notorious difference in MC activation seems to be related to disease evolution in L. mexicana infections, where in chronic infections of orchiectomized led to smaller lesions, as compared to sham mice (Figure 7).

Early neutrophil recruitment after L. mexicana infections have been associated with an increased parasite load and reduction of an efficient, protective immune response [35], indicating that L. mexicana exploits neutrophils to block the protective immune response by impairing the recruitment of dendritic cells to the infection site [36]. The results of our study now support the observation that neutrophils facilitate the L. mexicana infection and strongly suggests that MCs play an essential role during the early inflammation produced after L. mexicana and vector salivary gland proteins enter the host. Furthermore, inflammatory mediators (TNF-a, tryptase, and histamine) also favor recruiting an early wave of neutrophils, facilitating the infection. Besides, TNF-a, histamine, and tryptase can contribute to creating an immune-microenvironment that facilitates the establishment of Leishmania infection, priming MC to adopt an alternative activation phenotype (MC 2), or priming DCs to produce cytokines that favor a Th2-type immune response [37,38,39,40,41,42]. Furthermore, the evidence of an extensive release of tryptase and histamine by $\mathrm{MC}$ after a natural infection of $\mathrm{BALB} / \mathrm{c}$ mice with $\mathrm{L}$. major and Ph. duboscqi was observed by Sanchez-Garcia, 2018 [30]. Since the neutrophil-MC interaction facilitates L. mexicana infections, it is also essential to consider the gender in mouse models to describe the hormonal control of MC activation.

Throughout evolution, parasites have developed strategies to evade the host immune system and exploit it for the parasite benefit [43]. L. mexicana has developed a trans-regulation control of the host immune system, as evidenced by the fact that L. mexicana promastigotes show increased virulence and infection capacity in the presence of the male hormone dihydrotestosterone [23]. Furthermore, histamine release by MC is dose and genderdependent [44]. Our results now demonstrate that the severity of L. mexicana infection is influenced by the male sex hormones, which apparently regulate MC activation, promoting rapid neutrophil infiltration and the early release of inflammatory mediators, such as TNF-a, tryptase, and histamine. Thus, hormones are crucial during the development of the L. mexicana infection in a male host, where the infection is more severe than in females [7]. The cytokine CXCL8 or IL-8) is one of the most potent chemoattractant for guiding neutrophils through the tissue matrix until they reach sites of injury in mice. Some pieces of evidence indicated a hormonal regulation of several inflammatory mediators such as CXCL8. There is a possibility that the absence of male hormones delayed the arrival of neutrophils because of the control of the inflammatory mediators [45,46].

Finally, the slight mast cell increase in the ear of the orchiectomized mice seems to be related to the absence of male sexual hormones. This observation is in accordance with the significant enhancement of MC numbers reported in orchiectomized male mice, reaching similar numbers as those reported for females, which have been reported to have higher MC numbers [47,48]. Furthermore, the transient mastocytosis in both sham and gonadectomized mice after the stimuli with L. mexicana and vector SGL may be related to the antigenic challenge, since this transient mastocytosis was also observed in sham male BALB/c mice after a natural sand fly bite [30].

\section{Materials and Methods}

Ethics statement 
The protocol used in this study was approved by The Committee on Ethics and Use in Animal Experimentation of the Unidad de Medicina Experimental UNAM. The study was done following the guidelines of Mexican regulation for laboratory animals (NOM-062-ZOO-1999).

\subsection{Animals and experimental groups}

This study used four-week-old male BALB/c mice. The animals were singly housed in a controlled temperature $\left(22-24^{\circ} \mathrm{C}\right)$ and 12:12 light-dark conditions, receiving sterilized rodent diet and water ad libitum. The animals were organized into six groups of 5 animals each: 1) intact control, 2) infected control, 3) sham intact, 4) sham infected, 5) orchiectomized intact, and 6) orchiectomized infected. Orchiectomized male mice acquired the following characteristics: smooth hair, gained weight and became less aggressive. At necropsy, animals that presented reminiscence of testes were discarded.

\subsection{Surgical procedure}

Orchiectomy was performed using a mix of ketamine $(0.25 \%)$ and xylazine $(0.4 \%)$ for anesthesia. A small incision was made in the scrotum, the underlying muscle was cut, and the testes were extruded to the lower abdomen, ligated, and removed. For the sham-operated group, the testes were reinserted and non-ligated. Mice were operated under the day-light lamp to keep them warm and were monitored daily until the staples had self-removed.

\subsection{Parasite culture}

L. mexicana amastigotes were isolated from footpad lesions of infected BALB/c mice, as previously described [23-24]. Promastigotes were obtained by culturing isolated amastigotes at $26^{\circ} \mathrm{C}$ in culture medium 199, $\mathrm{pH} 7.2$, supplemented with $10 \%$ heat-inactivated fetal bovine serum (FBS), $100 \mathrm{U} / \mathrm{ml}$ penicillin $\mathrm{G}, 100 \mu \mathrm{g} / \mathrm{ml}$ streptomycin, and $2 \mathrm{mM}$ L-glutamine (all from Gibco-Life Technologies, Grand Island, NY, USA) and subpassaged during the logarithmic growth phase (days 3-4 of culture). For in vitro and in vivo infections, promastigotes at the stationary growth phase (day 5 of culture) were used. In this phase, most promastigotes have transformed into highly infectious metacyclic promastigotes. All promastigotes were cultured for no more than four in vitro sub-passages.

\subsection{Salivary Gland Collection}

Wild female sand flies flies were caught in Cunduacan, Tabasco, located in the southeast of Mexico, near the Grijalva river and Chontalpa subregion. The salivary glands of the sand fly species Bichromomyia olmeca olmeca (females) were obtained between 4 and 8 hours after collection. The flies were immobilized in cold PBS for about $2 \mathrm{~min}$. The dissected salivary glands were collected in phosphate-buffered saline (PBS) pH 7.2 and stored at $-80^{\circ} \mathrm{C}$ in batches per collecting day ( 5 pairs per batch). Lysates of the salivary glands were obtained by sonication and freeze-thaw cycles and centrifuged at $9900 \mathrm{xg}$ for $1 \mathrm{~min}$. The supernatant was collected and used immediately. Protein concentration was assessed by DC (DC protein Assay BIORAD 5000002). The average vector salivary gland protein concentration was $0.66 \pm 0.09 \mu \mathrm{g} / \mu \mathrm{l}$, corresponding to the pair of salivary glands of each sand fly.

\subsection{Infection procedure}

The infection procedure was performed as previously described by Dantas y col., 2009 [23]. Briefly, $0.06 \mu \mathrm{g} / \mu 1$ salivary proteins combined with 100 purified viable metacyclic L. mexicana promastigotes were injected into the dermis of both ears of each mouse. The evolution of the infection was monitored at 8, 24, 48, and 72 hours, and then at 4 and 8 weeks.

\subsection{Histology and immunohistochemistry}

The ears were gently flattened onto a piece of thick paper to avoid curling and cut by scissors into three equally sized fragments $(0.7 \times 1.6 \mathrm{~cm})$. Ears pieces were fixed in $4 \%$ paraformaldehyde in $0.1 \mathrm{M}$ Tris- $\mathrm{HCl}$ buffer $(\mathrm{pH} 7.2)$ for 24 hours. After carried out a conventional paraffin-embedded technique, the tissue sections were stained with hematoxylin-eosin (H\&E) or 2\% toluidine blue (198161, Sigma Aldrich) for histopathological analysis and MC identification, respectively. MC identification was made following the metachromatic principle [25]. TNF-a, histamine, and tryptase were assessed by immunohistochemistry in paraffin-embedded ear tissue sections. The immunohistochemistry procedure was as follows: tissue sections were de-waxed with xylene, rehydrated with $0.1 \mathrm{M}$ Tris- $\mathrm{HCl}$ buffer ( $\mathrm{pH} 7.2$ ), and transferred to plastic Coplin staining jars containing $0.1 \mathrm{M}$ citrate buffer ( $\mathrm{pH}$ 6.0) for antigen retrieval. Slides in the Coplin jar were then heated in a pressure cooker for $20 \mathrm{~min}$. at $200^{\circ} \mathrm{C}$ followed by $10 \mathrm{~min}$. at $100^{\circ} \mathrm{C}$. The slides were cooled in the jar at room temperature (RT) for $15 \mathrm{~min}$. and then transferred to $0.1 \mathrm{M}$ Tris- $\mathrm{HCl}$ buffer ( $\mathrm{pH}$ 7.2) until needed. After antigen retrieval, endogenous peroxidase was 
inhibited by incubation for $30 \mathrm{~min}$. at RT with 3\% hydrogen peroxide diluted in methanol. To reduce nonspecific background staining, slides were then incubated for 1 hour at RT in a solution containing $0.1 \mathrm{M}$ Tris- $\mathrm{HCl}$ buffer (pH 7.2), 2\% BSA, and 0.01\% Triton X-100. Slides were incubated overnight at $4^{\circ} \mathrm{C}$ with specific primary antibodies anti-TNF-a (1:50 anti-mouse N-19 sc 1350 Santa Cruz), anti-tryptase (1:100 Mast Cell Tryptase; anti-rabbit FL-275-Santa Cruz Biotech), or antihistamine (1:100 anti-rabbit ab78335 Abcam). After three washes, slides were incubated for $30 \mathrm{~min}$. with biotinylated secondary antibodies, either with anti-mouse IgG (diluted 1:50) (Jackson Immuno Research Laboratories cat \# 115035003) or with anti-rabbit IgG (diluted 1:50) (Sigma, USA) for 1 hour at RT. The avidin-biotin-HRP complex and 3,3'-diaminobenzidine were used, according to the manufacturer's instructions (Biocare Medical 901-DB801-010611). Finally, tissue sections were counterstained with hematoxylin (Sigma HHS16) for $1 \mathrm{~min}$. Only dark-brown colored cells with a visible nucleus and cytological features of MC were identified as positively stained mast cells. Control tissue sections were processed in the same manner, but primary antibodies were omitted.

\subsection{Statistics}

Nonparametric ANOVA ordinary one-way and multiple comparisons were used to test the statistical significance between groups. Significance was considered as $\mathrm{p}<0.001$. Tests were run by using GRAPH PAD PRISM 8 software.

Author Contributions: "Conceptualization, Sánchez-García, L.; Becker I and Morales-Montor, J. Methodology, Sánchez-García, L; Morales-Montor, J. Software, Sánchez-García, L; Validation, Becker Pérez-Torres, A. I. and Morales-Montor, J; Formal Analysis, Becker Pérez-Torres, A. I. Muñoz-Cruz, S and Morales-Montor.; Investigation, Sánchez-García, L.; Becker and I., Morales-Montor, J and Pérez-Torres, A; Resources, Becker, I. Writing - Original Draft Sanchez-García. Preparation, Sánchez-García, L and Morales-Montor, J; Writing - Review \& Editing, SánchezGarcía, L. Becker, I, Muñoz-Cruz, S. Pérez-Torres, A. Visualization, Becker, I., Muñoz-Cruz, S., Pérez-Torres, A. Funding Acquisition, Becker, I."

Institutional Review Board Statement: The protocol used in this study was approved by The Committee on Ethics and Use in Animal Experimentation of the Unidad de Investigación en Medicina Experimental UNAM number. Furthermore, the experimental study followed the guidelines of Mexican regulation for laboratory animals (NOM-062-ZOO-1999) and the Guide for the Care and Use of Laboratory Animals of the National Institute of Health, 8th Edition to ensure compliance with the established international regulations and guidelines.

Informed Consent Statement: Not applicable.

Data Availability Statement: All of the results were generated and included in this study.

Conflicts of Interest: The authors declare no conflict of interest.

\section{Acknowledgments / Funding}

This work was partially supported by research grants from the Programa de Apoyos a Proyectos de Investigación e Innovación Tecnológica (PAPIT) from Dirección General de Asuntos del Personal Académico (DGAPA), UNAM (Grant AG201221) and grant from CONACYT, Fronteras (6682) both to IB. 


\section{References}

1.- Krystel-Whittemore, M.; Dileepan, K.N.; Wood, J.G. Mast Cell: A Multi-Functional Master Cell. Front. Immunol. 2016, 6:620.

2.- Crivellato, E.; Ribatti, D.Th. e mast cell an evolutionary perspective. Biol Rev Camb Philos Soc. 2010, 85:347-360.

3.- Galli, S.; Tsai, M. Mast cells in allergy and infection: versatile effector and regulatory cells in innate and adaptive immunity. Eur. J. Immunol. 2010, 40: 1843-1851.

4.- da Silva, M.Z.E.; Jamur, M.C., Oliver, C. 2014. Mast Cell Function: A New Vision of an Old Cell. J Histochem Cytochem. 2014, 62(10) 69-738.

5.- Soman, N. A; St John, A.L. 2010. Mast cell-orchestrated immunity to pathogens. Nat Rev Immunol. 10(6): 440-4525.

6.- Harvima, I.; Nilsson, G. Mast cells as regulators of skin inflammation and immunity. Acta Derm Venereol. 2011. 91(6): 644-50.

7.- Villaseñor-Cardoso, M.I; Salaiza, N.; Delgado, J. Gutiérrez-Kobeh, L.; Pérez-Torres, A; Becker I. Mast cells are activated by Leishmania mexicana LPG and regulate the disease outcome depending on the genetic background of the host. Parasite Immunol. 2008, 30(8):425-34.

8.- Romão, P.R.T.; Da Costa Santiago, H.; Ramos, C.D.L.; De Oliveira, C.F.E.; Monteiro, M.C.; De Queiroz Cunha, F.; Vieira, L.Q. Mast cell degranulation contributes to susceptibility to Leishmania major. Parasite Immunology. 2009, 31:3:140-146.

9.- Kaye, P.M.; Beattie, L. Lessons from other diseases: granulomatous inflammation in leishmaniasis. Semin Immunopathol. 2016, 38:249-260.

10.- Andrade, B.B.; De Oliveira, C.I.; Brodskyn, C.C.; Barral, A.; Barral-Netto, M. Role of Sand Fly Saliva in Human and Experimental Leishmaniasis: Current Insights. Scan J Immunol. 2007, 66, 122-127.

11.- Rohousova, I.; Volf, P. Sand Fly: Effects on host immune response and Leishmania transmission. Folia Parasitologica. 2006, 53:161-171.

12.- Gomes, R.; Oliveira, F.; Teixeira, C.; Meneses, C.; Gilmore, D.C.; Elnaiem, DA.; Kamhawi, S.; Valenzuela, JV. Immunity to Sand Fly Salivary Protein LJM11 Modulates Host Response to VectorTransmitted Leishmania Conferring Ulcer-Free Protection. Soc Invest Dermatol. 2012, 132, 2735-2743.

13.- Muñoz-Cruz, S.; Togno-Pierce, C.; Morales-Montor, J. Non-reproductive effects of sex steroids: their immunoregulatory role. Curr Topic Med Chem. 2011, 11 (13), 1714-1727.

14.- Taneja, V. Sex Hormones Determine Immune Response. Frontiers in Immunology. 2018, 9:1931.

15.- Alexander, J. Sex differences and cross-immunity in DBA/2 mice infected with L. mexicana and L. major. Parasitol. 1988, 96(2): 297-302.

16.- Mock, B.A.; Nacy, C.A. Hormonal modulation of sex differences in resistance to Leishmania major systemic infections. Infect Immun. 1988. 56(12): 3316-3319. 
17.- Roberts, M.; Alexander, J.; Blackwell, J.M. Genetic analysis of Leishmania mexicana Infection in mice: single gene (Scl-2) controlled predisposition to cutaneous lesion development. J Immunol. 1990, 17(1): 89100.

18.- Satoskar, A.; Alexander J. Sex-determined susceptibility and differential IFN-gamma and TNF-alpha mRNA expression in DBA/2 mice infected with Leishmania mexicana. Immunol. 1995. 84: 1-4.

19.- Satoskar, A.; Al-Quassi, H.H.; Alexander, J. Sex-determined resistance against Leishmania mexicana is associated with the preferential induction of a Th1-like response and IFN-gamma production by female but not male DBA/2 mice. Immunol Cell Biol. 1988, 76(2): 159-166.

20.- Liu, L.; Wang, L.; Zhao, Y.; Wang, Y.; Wang, Z.; Qiao, Z. Testosterone attenuates p38 MAPK pathway during Leishmania donovani infection of macrophages. Parasitol Res. 2006. 99: 189-193.

21.- Zhang, H.; Zhao, J.; Wang, P.; Qiao, Z. Effect of testosterone on Leishmania donovani infection of macrophages. Parasitol Res. 2001, 87(8): 674-676.

22-. Yin, G.; Guo, Z.; Yin, K.; Zhao, J. Effect of testosterone on Leishmania donovani infection levels of murine bone marrow-derived macrophages. Zhongguo Ji Sheng Chong Xue Yu Ji Sheng Chong Bing Za Zhi. 1988, 16(4): 251-255.

23.- Sánchez-García, L.; Wilkins-Rodriguez, A.; Salaiza-Suazo, N.; Morales-Montor, J.; Becker, I. Dihydrotestosterone enhances growth and infectivity of Leishmania mexicana. Parasite Immunol. 2018, 40(3), e12512. doi:10.1111/pim.12512.

24.- Dantas, C.; Carvalho de Souza, C.; França, C.; Quercia, V.; Crocco, L.; Esteves, A. Histopathology of Leishmania major infection: revisiting L. major histopathology in the ear dermis infection model. Mem Inst Oswal Cruz. 2009, 104:918.

25.- Sridharan, G.; Shanka, A.A. Toluidine blue: A review of its chemistry and clinical utility. J Oral Maxillo Pathol. 2012. 16:251-255.

26.- von Stebut, E. Immunology of cutaneous leishmaniasis: the role of mast cells, phagocytes and dendritic cells for protective immunity. Eur. J. Dermatol. 2007, 17:115-122. DOI: 10.1684/ejd.2007.0122.

27.- Maurer, M.; Lopez Kostka, S.; Siebenhaar, F.; Moelle, K.; Metz, M.; Knop, J.; von Stebut E. Skin mast cells control T cell-dependent host defense in Leishmania major infections. FASEB J. 2006, 20:2460-2467. ç

28.- von Stebut, E.; Metz, M.; Milon, M.; Knop, J.; Maurer, M. Early macrophage influx to sites of cutaneous granuloma formation is dependent on MIP- $1 \alpha / \beta$ released from neutrophils recruited by mast cell-derived TNF $\alpha$. The FASEB Blod. 2006, 101(1):210-5

29.- Bidri, M.; Vouldoukis, I.M. Evidence for direct interaction between mast cells and Leishmania parasites. Parasite Immunol. 1997， 19:475-83.

30.- Sánchez-García, L. Estudio de la Regulación que Ejercen la Testosterona y Extractos Salivales de Lutzomyia olmeca sobre las Células Cebadas y su Asociación con la Leishmaniasis. PhD Thesis.

Universidad Nacional Autónoma de México. México. D.F. 2018.

31.- Lockard, R.D.; Wilson, M.E.; Rodríguez, N.E. Sex-Related Differences in Immune Response and Symptomatic Manifestations to Infection with Leishmania Species. J Immunol Res. 2019, 1-14. 
32.- Vom Steeg, L.G.; Klein, S.L.; Sex steroids mediate bidirectional interactions between hosts and microbes. Hormon Behavior. 2017, 88; 45-51.

33.- Klein, S L.; Flanagan, K.L. Sex differences in immune responses. Nat Review Immunol. 2016,16(10) 626-638.

34.- Hurrel, P.B.; Beumann, M.; Heyde, S.; Regli, IB.; Müller, J.A.; Tachinni-Cottier F. Frontline Science: Leishmania mexicana amastigotes can replicate within neutrophils. J Leukoc Biol. 2017, 102(5):1187-1198.

35.- Ribeiro-Gomes, F.L.; Sacks, D. The influence of early neutrophil-Leishmania interactions on the host immune response to infection. Front Cell Infect Microbiol. 2012, 2:59.

36.- Hurrell, B.P.; Schuster, S.; Grün, E.; Coutaz, M.; Williams, R.A.; Held W, Bernard Malissen, B.; Malissen, M.; Yousefi, S.; Simon, H.U; Müller, J.M.; Tacchini-Cottier, F. Rapid Sequestration of Leishmania mexicana by Neutrophils Contributes to the Development of Chronic Lesion. PLoS Pathog. 2015, 11(5): e1004929.

37.- Soong, L. Modulation of Dendritic Cell Function by Leishmania Parasites. J Immunol. 2008, 180(7):4355-60.

38.- Meyer, C.M.; Creer, H.M.; McHowat, J. Potential role for mast cell tryptase in recruitment of inflammatory cells to endothelium. Amer. J. Physiol. Cell. Physiol. 2005, 289:C1485-C91.

39.- Pós, Z.; Müller, K.; Novák, I.; Buzás, E.; Solbach, W.; Falus, A.; Laskay, T. Different patterns of the Lhistidine decarboxylase (HDC) gene expression in mice resistant and susceptible to experimental cutaneous leishmaniasis. Inflamm. Res. 2004, 53:38-43.

40.- Qi, H.; Popov, V.; Soong, L. Leishmania amazonensis-dendritic cell interactions in vitro and the priming of parasite-specific CD4(+) T cells in vivo. J Immunol. 2001, 5;167(8):4534-42.

41.- Mazzoni, A.; Young, H.; Spitzer, J.; Visintin, A.; Segal, D. Histamine regulates cytokine production in maturing dendritic cells, resulting in altered T cell polarization. J. Clin. Invest. 2001, 108:1865-1873.

42.- Caron, G.; Delneste, Y.; Roelants, E.; Duez, C.; Bonnefoy, J.; Pestel, J. Jeannin, P. Histamine polarizes human dendritic cells into Th2 cell promoting effector dendritic cells. J. Immunol. 2001, 167:3682-3686.

43.- Bernard, G. Within-Host Parasite Dynamics, Emerging Trade-Off, and Evolution of Virulence with Immune System. Evolution. 2003, 57(7): 489-1497.

44.- Muñoz-Cruz, S.; Mendoza-Rodriguez, Y.; Nava-Castro, K.; Yepez-Mulia, L.; Morales-Montor, J. Gender-related Effect of Sex Steroides on Histamine Released and FceRI Expression in Rat Peritoneal Mast Cells. J Immunol Res. 2015, Article ID 351829. 10pages.

45.- Poili, P.; Jensen, A.L.; Weaver, L.K.; Wira, C.R.; Guye, P.L. Estradiol Attenuates LipopolysaccharideInduced CXC Chemokine Ligand 8 Production by Human Peripheral Blood Monocytes. J Immunol 2007, 179:6284-6290.

46.- Evans, J.; Salamonsen, L.A. Decidualized Human Endometrial Stromal Cells Are Sensors of Hormone Withdrawal in the Menstrual Inflammatory Cascade. Biol Rep. 2014, 90(1):14, 1-12.

47.- Shirama, K.; Kohda, M.; Hokano, M. Effects of endocrine glands and hormone replacement on the mast cell count of the Harderian gland of mice. Acta Anat. 1988, 131(4):327-31. 
48.- Payne, A.P.; Mc Gadey, J.; Johnston, H.S.; Moore, M.R.; Thompson, G.G. Mast cells in the hamster Harderian gland: sex differences, hormonal control and relationship to porphyrin. J. Anat. 1982, 135:3:451461. 